\title{
Una rúbrica para evaluar y mejorar los proyectos de aprendizaje servicio en la universidad
}

\author{
Laura Campo Cano laura.campo80@gmail.com
}

Fundació Jaume Bofill, España

\section{Resumen}

El artículo plantea estudiar qué aspectos tienen que tener las propuestas de aprendizaje servicio en educación superior para tener una alta calidad y elaborar una herramienta de evaluación para esta calidad adecuada al contexto universitario español. Planteamos una rúbrica de autoevaluación de la calidad de proyectos de aprendizaje servicio en educación superior que esté diseñada para ayudar al profesorado a ajustar y mejorar el desarrollo y el enfoque de los proyectos de aprendizaje servicio que estén llevando a cabo o quieran diseñar. La rúbrica está estructurada en diez categorías fruto del análisis de los puntos clave considerados por los expertos como aspectos relevantes de la calidad de los proyectos de aprendizaje servicio en su contexto educativo.

\section{Palabras clave}

Aprendizaje servicio, calidad educativa, evaluación de proyectos, rúbrica, educación superior. 


\title{
A rubric for assessing and improving service learning projects at university
}

\begin{abstract}
The objective of this paper is to study which aspects must be present in a service learning proposal, in the context of higher education, in order to ensure a high quality outcome, and to create a tool to evaluate said outcomes. Therefore, the objective is to use a self-evaluating rubric to measure the quality of service learning proposals in higher education, and to assist faculty members to make necessary adjustments to improve the development and focus of programs that they are managing or creating. The rubric has ten categories based on the analysis of key points which experts agree should exist in order to ensure a quality service learning program in an educational context.
\end{abstract}

\section{Palabras clave}

Learning-Service, Educational Quality, Project Evaluation, Rubric, Higher Education. 


\section{Introducción}

En el texto se presentan los aspectos que se consideran relevantes para los proyectos de aprendizaje servicio en la universidad. El objetivo es estudiar los elementos que dan calidad a los proyectos de aprendizaje servicio y proponer una herramienta para la evaluación de ésta, una rúbrica para evaluar la calidad de proyectos. La elaboración de la rúbrica se ha construido a partir de un diálogo entre la teoría y la voz del profesorado experto en proyectos de aprendizaje servicio. Se ha analizado la literatura del tema, se han realizado siete entrevistas en profundidad a profesorado experto y con experiencia; se han realizado dos grupos de discusión (con 10 y 5 participantes en cada uno); y doce entrevistas informales a expertos internacionales. Finalmente, la propuesta de la rúbrica fue revisada, una vez construida, por cinco expertos en la temática.

Entendemos la evaluación como la valoración, a partir de criterios y referencias preespecificadas, de la información técnicamente diseñada y sistemáticamente recogida y organizada, sobre factores relevantes que integran los procesos educativos para facilitar la toma de decisiones de mejora (Pérez Juste, 2006, p.32).

Consideramos la evaluación como una herramienta de mejora continua, una herramienta formativa, siendo ésta su función principal. Esta función de mejora representa una visión amplia de la evaluación en la que se evalúan todos los elementos del proyecto, no sólo los contenidos académicos; los evaluadores pueden ser todos aquellos que tengan información relevante; se evalúa en diferentes momentos a lo largo del desarrollo del proyecto y de manera continuada.

El carácter formativo de la evaluación hace que ésta se considere como un elemento inherente al proceso de enseñanza y aprendizaje y como un instrumento al servicio de este proceso. Las situaciones y actividades que se utilizan para identificar y valorar qué han aprendido los estudiantes constituyen un punto de unión entre las propuestas del profesorado y los aprendizajes que realizan los alumnos (Coll, Martín y Onrubia, 2001).

Así, entendemos la evaluación como un proceso de mejora de los proyectos de aprendizaje servicio, una reflexión para saber hacia dónde ir, para tener una guía de mejora.

\section{La calidad en los proyectos de aprendizaje servicio.}

A continuación vamos a ver qué aspectos se consideran relevantes para la calidad de los proyectos de aprendizaje servicio en la universidad. En este sentido, consideramos que un proyecto es de calidad cuando reúne las características que permiten lograr los objetivos inherentes al proyecto y los lleva a cabo con éxito. 
Proponemos a continuación diez criterios de calidad a tener en cuenta para valorar la calidad de los proyectos de aprendizaje servicio.

\section{Un enfoque de aprendizaje profundo}

Los enfoques de aprendizaje describen los motivos o intenciones para aprender y las estrategias utilizadas ante una tarea de aprendizaje. Los enfoques de aprendizaje resultan de la relación entre los motivos para aprender de un estudiante y las estrategias, propuestas e individuales, para abordar dicho aprendizaje.

El enfoque profundo afronta la tarea hacia el significado y la comprensión. El estudiante busca significado en los aprendizajes de un modo global integrándolo realmente en sus conocimientos previos, interaccionando críticamente con los contenidos. En este caso, el interés y la motivación son intrínsecos y se concibe el aprendizaje como una satisfacción personal. Es una concepción del aprendizaje compleja, sofisticada, donde las estrategias que se utilizan están basadas en el interés del estudiante hacia la tarea y se relacionan los diversos componentes de la tarea entre sí, integrándolas en un conjunto (Hernández Pina y otros, 2005, p.11).

La concepción del aprendizaje que tienen los estudiantes con un enfoque profundo está relacionada con una idea cualitativa del aprendizaje. Es cierto que los estudiantes muestran, de antemano, un enfoque de aprendizaje u otro, pero también viene determinado por el tipo de estrategias que se proponen al estudiantado.

Las propuestas de aprendizaje servicio, entre otras prácticas que procuran objetivos parecidos, se basan en una concepción del aprendizaje profundo procurando el significado y no el hecho de aprender para reproducir. Las prácticas de aprendizaje servicio enfatizan el aprendizaje facilitador de significado y el comprender mejor y ver las cosas de manera diferente (Martínez y Puig, 2011). El desarrollo de una práctica de aprendizaje servicio de calidad debería implicar un aprendizaje riguroso, vinculado estrecha y simultáneamente a un acción solidaria planificada que procura impactar de forma positiva sobre la vida de una comunidad (Herrero, 2010).

Los aprendizajes en los proyectos de aprendizaje servicio deben ser planificados, sistemáticos, conscientes $y$, en la medida de lo posible, vinculados a los contenidos curriculares (Puig y otros, 2007). En las actividades de aprendizaje servicio, los aprendizajes ayudan a comprender la complejidad de la realidad donde se va a actuar (Rubio, 2009). 


\section{El desarrollo de competencias}

Se considera que el desarrollo de competencias implica una combinación compleja de conocimientos, técnicas, habilidades y valores que resulta crítica para hacer bien aquello que se le exige en circunstancias en las que se encuentre mientras realiza una actividad profesional (De Miguel, 2005).

Por su sentido globalizador, el aprendizaje servicio se convierte en una excelente metodología para adquirir competencias, integrando capacidades, habilidades, conocimientos y valores que se movilizan para resolver situaciones reales (Rubio, 2009). El escenario adecuado para el aprendizaje competencial -contextualización, interdisciplinariedad, aplicación y experimentación, incidencia social y comunitaria y reflexión- es el escenario propio de los proyectos de aprendizaje servicio (Palos, 2011).

El aprendizaje servicio nos permite proponer situaciones en las que tenga que haber comprensión, transferencia de conocimientos, resolución de problemas, reflexión crítica en un contexto real y complejo. Son situaciones en las que el alumno demostrará si es competente resolviendo la situación real propuesta.

Los tipos de competencias que distinguimos en los proyectos de aprendizaje servicio en la universidad son competencias transversales o genéricas, competencias profesionales, competencias específicas y competencias de investigación.

Sobre las competencias genéricas cabe destacar que se valoran los proyectos de aprendizaje servicio como un buen espacio para el trabajo en equipo ya que prácticamente no encontramos propuestas de trabajo individual. Otras competencias genéricas son las habilidades sociales, la empatía, la creatividad, la resolución de problemas y el liderazgo. También la explicitación de los proyectos de aprendizaje servicio como un espacio para aprender valores ciudadanos y personales llevándolos a la práctica, viviéndolos.

Sobre las competencias profesionales, aquellas que atañen a la profesión en general y que podríamos enmarcar en las competencias genéricas instrumentales, vemos que los proyectos de aprendizaje servicio se consideran un buen espacio para desarrollarlas. Además se aprenden competencias profesionales que difícilmente se aprenden con otro tipo de proyectos. Destacamos que en los proyectos de aprendizaje servicio se pone en relieve la importancia de conocimientos profesionales interdisciplinares.

Las competencias específicas son aquellas propias de cada área temática. Son cruciales para cualquier titulación porque están específicamente relacionadas con las disciplinas académicas y son las que confieren identidad. Los proyectos de aprendizaje servicio se refieren a las competencias curriculares. No siempre es fácil vincular los proyectos al 
currículum, a veces resulta un poco forzado, pero si es posible, deberíamos tener el trabajo de las competencias curriculares como objetivo. En general, valoramos la importancia de los aprendizajes más curriculares como un elemento de calidad de las propuestas de aprendizaje servicio

Finalmente, y de manera global, valoramos como fundamental que los proyectos de aprendizaje servicio hagan hincapié en la formación académica de los estudiantes.

También queremos distinguir las competencias de investigación porque nos parece que la universidad es el espacio que debería promover las competencias propias de la indagación y la investigación y los proyectos de aprendizaje servicio pueden hacer hincapié en este tipo de competencias.

\section{El nivel de participación de los estudiantes}

Participar de manera real en sociedades democráticas como la nuestra significa no recluirse en los asuntos privados, sino participar, de un modo u otro, en la construcción de una sociedad justa (Cortina, 1997). De esta manera, se apuesta por una participación compleja más allá de las individualidades. La participación se define en relación a lo comunitario, al empoderamiento personal, a la capacidad de transformar la comunidad de los individuos y del colectivo.

Tomando como referencia la clasificación de Trilla y Novella (2001) sobre la participación infantil vemos en los proyectos de aprendizaje servicio, como mínimo tres tipos de participación. Son proyectos participativos en los que los estudiantes hacen acciones y actividades en la comunidad. Este tipo de participación está asegurada y es una participación simple. En proyectos educativos como los de aprendizaje servicio se pueden consultar a los estudiantes múltiples aspectos como las necesidades sobre las que trabajar, las entidades con las que conectar, las acciones a llevar a cabo, el papel de los propios estudiantes y de los receptores del servicio, aspectos sobre el desarrollo del proyecto, la evaluación, etc. Esta es una participación consultiva. Otro tipo de participación es aquella en que los estudiantes pueden intervenir, en mayor o menor medida, en el propio proyecto (diseño, organización, gestión, etc.). En la participación proyectiva el estudiante se convierte en un agente que hace el proyecto suyo, no se limita a ser un usuario ni a opinar desde fuera, sino que interviene directamente en el proyecto. Es un tipo de participación que requiere compromiso y corresponsabilización. Los proyectos de aprendizaje servicio son un buen espacio para proponer situaciones donde llevar a cabo la participación proyectiva. Se puede ampliar a todo el proceso o solo a una parte de él. Este tipo de participación es frecuente y recomendable en proyectos de aprendizaje servicio en educación superior. 


\section{La evaluación del proyecto}

La evaluación ofrece información para comprender mejor los fenómenos educativos, nos ayuda entender las prácticas educativas. También nos ayuda a desarrollar procesos y estrategias educativas adecuadas a nuestros estudiantes y a su contexto. La evaluación comprueba la utilidad de los procedimientos propuestos, la adecuación de los objetivos y sus resultados para mejorar las propuestas de programas, objetivos, metodologías, etc.

Las actividades y los instrumentos de evaluación, que podrán ser diversos, tendrán que ser coherentes con los elementos del proyecto educativo y con los objetivos que éste se plantea. Se puede recoger información para evaluar a través de la observación de los participantes, de cuestionarios, entrevistas, textos reflexivos, reflexiones orales, debates, portafolios.

Una vez recogida la información, para evaluarla, se debe valorar a partir de unos criterios y referencias previamente definidos y compartidos por todos los participantes. Los criterios de evaluación permiten atribuir valor a las actividades, los trabajos, las actitudes de los participantes del programa (Pérez Juste, 2006). Tener los criterios planificados desde el planteamiento de la propuesta ayuda a hacer valoraciones con mayor grado de objetividad y a orientar las decisiones que se deban tomar para la mejora del programa en cualquiera de sus aspectos.

El valor que le damos a la evaluación es como proceso de mejora y de búsqueda de alternativas de mejor calidad. Así, la evaluación que planteamos en los proyectos de aprendizaje servicio no es estática, sino dinámica y continuada. Proponemos una evaluación que se da a lo largo de todo el proyecto, en la planificación y la ejecución del mismo con el objetivo que ésta nos proporcione una retroalimentación que permita ir haciendo los ajustes necesarios. La evaluación será una parte más a planificar en el desarrollo del proyecto.

La evaluación se considerará multifocal y se tendrá en cuenta la dimensión pedagógica y la dimensión social de los proyectos de aprendizaje servicio. Así, se evaluaran los aprendizajes, cómo se ha llevado a cabo el servicio y la propia experiencia en general.

El profesorado podrá hacer dos tipos de evaluación: una con los estudiantes y otra que el profesorado y los responsables del proyecto. Con los estudiantes se puede hacer balance de los resultados del servicio para valorar cómo ha ido el compromiso que se había adquirido con la comunidad, medir los resultados posibles, valorar condiciones que han ido surgiendo, etc. Con los estudiantes también tendremos que dedicar un espacio y un tiempo a hacer una reflexión y un balance de los propios aprendizajes tanto de conceptos como de procedimientos, habilidades y actitudes y valores. Ya en la 


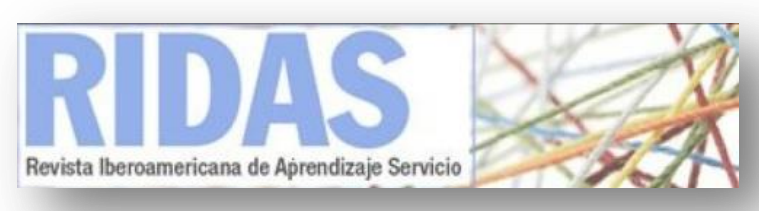

finalización del proyecto cabe un espacio para valorar qué posibilidades nos brinda el proyecto una vez finalizado, para valorar la proyección y las perspectivas de futuro (Puig y otros, 2007).

Otro tipo de evaluación es aquella que hará el profesorado. Además de evaluar el grupo y a cada estudiante, el profesorado debería dedicar un espacio a valorar el trabajo en red con las entidades, la evaluación de la experiencia en general y una autoevaluación de sus funciones y sus propuestas (Puig y otros, 2007). En estas reflexiones generales sobre la experiencia no deberíamos obviar las voces de los responsables de las entidades, de los beneficiarios del servicio prestado ni de los alumnos.

Consideramos que como propuesta de alta calidad deberíamos pensar en que la mayoría de participantes evalúen las diferentes partes del proyecto. Los aprendizajes que han adquirido los estudiantes pueden ser valorados por: el profesorado que los ha planificado, los responsables de las entidades que los han visto en ejecución, los beneficiarios del servicio que han sacado provecho de estos y por los propios estudiantes a modo de autoevaluación. El servicio debería ser valorado por: los estudiantes que lo han llevado a cabo, los responsables de las entidades que han hecho seguimiento, el profesorado que lo ha coordinado junto con la entidad y los beneficiarios que lo han recibido. La experiencia en general, como proyecto también debería ser valorada por todos los participantes. De esta manera podremos tomar decisiones fundamentadas para hacer cambios, mejoras, continuar con el proyecto, cancelarlo o adaptarlo a nuevas situaciones en mejores condiciones.

\section{El seguimiento académico en la entidad}

Los proyectos de aprendizaje servicio suelen llevarse a cabo en dos instituciones: la primera, la académica, y la segunda, la entidad donde se lleva a cabo el servicio. En este binomio, las dos tienen un peso parecido, las dos son activas y tienen un papel definitorio en el desarrollo del proyecto. No nos deberíamos imaginar una entidad que simplemente acoge a los estudiantes pero que no ha podido diseñar conjuntamente el proyecto, explicitar sus verdaderas necesidades, valorar el papel real de los estudiantes en su entidad, etc. Además, como parte del aprendizaje también tendrá lugar en la entidad, ésta tendrá un peso en la evaluación. Este último tema lo abordaremos más adelante cuando expliquemos la categoría de la evaluación.

En los proyectos de aprendizaje servicio encontraremos diversos tipos de entidades donde los estudiantes desarrollan el servicio: organizaciones no gubernamentales, otros espacios universitarios, instituciones educativas, administraciones locales. Así, en este tipo de proyectos no podemos olvidar el referente en la entidad, aquél que 
también es tutor de nuestro estudiante y hace seguimiento en el espacio donde se lleva a cabo el servicio.

Se propone que haya una explícita coordinación con el responsable de la entidad donde sus estudiantes llevan a cabo el servicio, se debe de facilitar un buen espacio de intercambio y comunicación; los objetivos del proyecto y el desarrollo concreto del mismo deben haberse compartido entre los participantes -profesorado, estudiantes y responsables de entidades-; el profesorado debe seguir el proceso de aprendizaje del alumno y debe facilitar recursos para que pueda avanzar; el profesorado debe estar atento a posibles dificultades académicas de los estudiantes pero también y especialmente a las que se puedan dar durante el desarrollo del servicio y ayudar a resolverlas; debe facilitar las condiciones para que los responsables de la entidad se impliquen en el desarrollo del proyecto. En general, el tutor debe velar por el buen desarrollo del proyecto.

\section{La transdisciplinariedad de las propuestas}

La transdiciplinariedad propone una nueva actitud frente a cuestiones como la multiplicidad de los saberes de las distintas disciplinas para hacerlas menos fragmentadas, más conectadas, más holísticas. Los límites de las diversas disciplinas se difuminan, se hacen porosas para conectarse con las demás. Se tiene como objetivo entender el mundo en su globalidad, de manera integrada.

Muchos de los retos actuales tienen una naturaleza compleja que deberíamos abordar desde una perspectiva transdisciplinar. Necesitaremos prácticas de aprendizaje y quehacer holístico que transcienda de la fragmentación del saber y el conocimiento pero no lo ignore. $Y$ el aprendizaje servicio nos ofrece la oportunidad de vivir la complejidad.

En algunos proyectos de aprendizaje servicio se han incorporado varias disciplinas para innovar y dar mayor calidad a las experiencias. La colaboración con otras disciplinas también puede ser una aportación y ofrecer una mirada más externa. En este sentido, asumir propuestas transdisciplinares de aprendizaje servicio puede conllevar o facilitar una organización determinada para la institución académica.

Cuando estudiantes de cualquier ámbito se complementan con otros para cubrir unas mismas necesidades reales supone un nivel de complejidad muy importante que corresponde a la transdisciplinariedad donde la relación entre disciplinas va más allá de cada una de ellas para resolver un reto compartido. 


\section{El impacto y la proyección social}

Cuando se habla de impacto en los proyectos de aprendizaje servicio se plantean cuestiones relacionadas básicamente con la calidad de la oferta educativa y en relación a los estudiantes. Así, el aprendizaje servicio impacta en el aprendizaje y mejora la comprensión y la aplicación de los conocimientos, en el desarrollo del interés por indagar y del pensamiento reflexivo y crítico, en la percepción de posibilidades de cambio social, en el desarrollo personal e interpersonal y en el desarrollo de prácticas de ciudadanía (Eyler y Giles, 1999). También se ha mostrado que hay impactos en el desarrollo de las competencias para la inserción en el mundo del trabajo, en la formación ética y en la participación social y política de los estudiantes que participan en proyectos de aprendizaje servicio (Tapia, 2008). Otro impacto que se puede ver a largo plazo que tiene un impacto en la comunidad $y$, no solo en el estudiante, es que se está demostrado que los que participan en actividades de aprendizaje servicio o de voluntariado tienden a participar en entidades sociales cuando son adultos (Tapia, 2007).

También podemos reflexionar sobre si las necesidades han sido cubiertas y en qué medida y también sobre si el proyecto ha creado nuevos espacios de participación y de reflexión, ha facilitado la asociación o algún tipo de cambio político, ha ayudado a formar redes para producir nuevos cambios, o si ha impactado en otros colectivos de manera indirecta. Esta mirada hacia la comunidad nos obliga a hacer especial hincapié sobre ella, darle identidad y un peso específico en el diseño del proyecto. Hacer que la comunidad sea protagonista para valorar e intentar sacar el máximo de provecho a la propuesta de aprendizaje servicio que hagamos.

En general se comparte la idea de que las necesidades que se deben cubrir son en un entorno cercano y que para ello se debe analizar bien la situación que nos rodea. Se considera que los estudiantes se implicaran más y verán más claramente los resultados, conocer la comunidad más cercana es la manera de establecer vínculos con ella.

Acerca del impacto social, el proyecto tiene que tener en cuenta la sostenibilidad de los cambios que proporciona la intervención y ofrece herramientas para que la comunidad siga con las mejoras propuestas. Para Tapia (2007) éste es un criterio de calidad: la transferencia de saberes y de tecnologías a la comunidad. Un buen proyecto de aprendizaje servicio pasa, no sólo por lo que los estudiantes puedan hacer a lo largo del proyecto, sino por lo que la comunidad pueda seguir haciendo en el mediano y largo plazo con las herramientas que se le hayan ofrecido. 


\section{El trabajo en red}

Consideramos que las redes son estructuras que se muestran flexibles, capaces de adaptarse a situaciones nuevas y cambiantes. Las redes educativas, por su estructura y organización suponen un cambio cualitativo respecto a la manera de organizarse anteriormente ya que las redes permiten movilizar el conocimiento y adaptarse a diversas situaciones propias del momento actual caracterizado por la diversidad, la complejidad, el cambio y la incertidumbre.

En este contexto los proyectos de aprendizaje servicio se conciben como creadores de la red. Consideramos que crean red educativa porque las instituciones educativas se unen con las entidades sociales, las organizaciones no gubernamentales, la administración, etc. La unión con las diferentes entidades o instituciones se hace creando lazos de partenariado. La creación de los lazos de partenariado supone conocerse, decidir colaborar, acordar un proyecto, aplicarlo, evaluarlo y decidir nuevas acciones. Cada una de las partes, contará con un tiempo y un ritmo determinado que deberemos respetar. A menudo, las entidades sociales, por su estructura, tienen un ritmo más lento y deberemos ponernos en el lugar del otro para comprender su situación. En este sentido, podemos afirmar que el aprendizaje servicio no se puede impulsar fácilmente en solitario y afirmamos que la necesidad de unir en un solo proyecto útil e interesante para ambas partes supone construir relaciones de partenariado entre las instituciones implicadas (Puig, Campo y Climent, 2011).

En ocasiones, las relaciones de partenariado empiezan con contactos personales y de manera bastante natural. Otras veces se buscan los contactos a partir de referencias. Sea de una forma u otra, los contactos personales aún tienen un fuerte peso en el momento de buscar aliados para llevar a cabo proyectos de aprendizaje servicio. Se tiene que crear una red social importante, ser hábil en el momento de contactar y poder convencer en el "cara a cara" a las personas de la entidad.

En un nivel de mayor complejidad y calidad, los proyectos se pueden unir con otros similares para compartir conocimientos, experiencias, recursos, etc. creando una red de proyectos de aprendizaje servicio de un tipo determinado.

\section{Campo profesional en los proyectos de aprendizaje servicio}

En los proyectos de aprendizaje servicio los estudiantes aprenden habilidades y experiencias valiosas para el desarrollo de su vida profesional. La vivencia que suponen los proyectos de aprendizaje servicio hace que algunos estudiantes cambien su orientación laboral hacia ámbitos laborales más sociales donde se valore el servicio al otro (Eyler y Giles, 1999). 


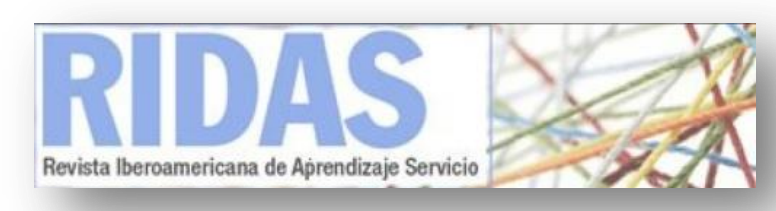

En este sentido, los proyectos de aprendizaje servicio amplían la conciencia y las opciones vocacionales, mejoran las competencias profesionales, se ayuda a crear mayor comprensión de la ética del trabajo y se preparan mejor para el mundo del trabajoi. Relacionado también con estas cuestiones vocacionales participar en proyectos de aprendizaje servicio beneficia actitudes positivas y realistas en torno al trabajo (Arratia, 2008).

Podemos ver que hay un impacto en el desarrollo de competencias para la inserción del mundo del trabajo. Las experiencias de aprendizaje servicio contribuyen a superar la brecha entre teoría y práctica. Las prácticas de aprendizaje servicio pueden contribuir a competencias valoradas en el mundo laboral como el trabajo en equipo, habilidades comunicativas, la iniciativa, el liderazgo, etc. (Tapia, 2008). Además podemos deducir que los estudiantes involucrados en programas de aprendizaje servicio adquieren habilidades como autoconocimiento de las propias destrezas y preferencias profesionales, actitudes y destrezas positivas dirigidas a la búsqueda de trabajo.

Esta característica que da calidad a los proyectos se considera especialmente importante en aquellos estudios menos sociales. Se valora que puede haber un cambio de mirada profesional en positivo. Además, los estudiantes tienen la oportunidad de trabajar de manera real con profesionales de diferentes disciplinas. Las situaciones de trabajo real con otros profesionales que tienen los estudiantes a lo largo de los estudios es realmente muy limitada o escaso en la mayoría de propuestas de aprendizaje. Es una excelente manera de comprender una visión realista y compleja de un posible campo profesional en el que desarrollar en el futuro la vida laboral.

\section{La institucionalización}

La institucionalización del aprendizaje servicio tiene sentido en una universidad que considera que mediante la educación es posible la transformación de la sociedad en una más digna, inclusiva, cohesionada y equitativa. Una institución que considera importante tanto la preparación para el mundo laboral como la formación para una ciudadanía activa (Martínez, 2008). En este modelo la universidad se reconoce como parte del conjunto de la comunidad, no está aislada ni tampoco supeditada a las demandas de organizaciones o empresas. La institucionalización de las propuestas de aprendizaje servicio es una opción ideológica y política de la universidad. Estas actividades tienen sentido en una universidad comprometida con la sociedad, que considera que debe formar a los estudiantes como ciudadanos

Resultados de numerosos estudios revelan que el apoyo de las administraciones resulta crucial para desarrollar y hacer sostenibles los programas de aprendizaje servicio (Jeandron y Robinson, 2010). Un cierto grado de institucionalización resulta 
necesaria si se quiere avanzar en la consolidación de los proyectos de aprendizaje servicio.

La institucionalización se concibe como una manera de replicar proyectos, de crecer, de llegar a la mayoría, en definitiva, de evitar ir hacia atrás o estancarse. Es también una buena estrategia para hacer los proyectos más sostenibles porque el profesorado se siente mucho más seguro de llevar a cabo este tipo de proyectos y se siente más reconocido. Además no deja de ser una concreción de la responsabilidad social de las universidades. La institucionalización también debería exigir unos mínimos de calidad para los proyectos de aprendizaje servicio. De alguna manera, la institución debe marcar unos mínimos y unas líneas estratégicas de hacia dónde se quiere ir. Debería de ser una validación inicial, de seguimiento y final en las que se asegure que los requisitos se cumplen a lo largo de todo el proyecto.

En definitiva, consideramos que la universidad debería crear las condiciones adecuadas para facilitar y hacer sostenibles estos proyectos.

Sobre el reconocimiento consideramos que fuera positivo que los proyectos de aprendizaje servicio tuvieran una mayor presencia en los planes de estudio para que no solamente se impulsara en estudios sociales donde resulta más sencillo y directo sino en cualquier tipo de estudios. Fuera positivo que la universidad encontrara alguna forma especial de reconocimiento. En este sentido, para facilitar la organización del desarrollo de estos proyectos es interesante que forme parte de un programa que haya sido aprobado por el consejo de gobierno de la universidad. En este caso se deben fijar unos mínimos que deben de cumplir los programas de aprendizaje servicio y se pueden fijar cuestiones generales como temas de seguros de los estudiantes o de propiedad intelectual, de convenios entre entidades que participan en el proyecto, etc.

Por otro lado, también valoramos muy especialmente contar con recursos para destinar a la coordinación de estos proyectos. Al profesorado no le resulta fácil tejer relaciones con las entidades y hacer el seguimiento de manera detallada. Así, que exista un espacio de coordinación con alguna persona con dedicación específica es un recurso muy potente. Una de las propuestas para hacer más sostenible este espacio de coordinación es resolver algunas cuestiones prácticas a través de material escrito de manera que la información pueda estar colgada en la red para que llegue a todo el profesorado de manera eficaz. Se trata de construir algunas herramientas donde se resuelvan las preguntas clave y se den indicaciones de cómo llevar a cabo una propuesta de aprendizaje servicio.

Para que los programas de aprendizaje servicio tengan éxito deben desarrollarse en un ambiente positivo en el que haya recursos de apoyo y espacios de celebración. La institución juega un papel importante en la consolidación de un clima como éste 


\section{RDAS}

(Jeandron y Robinson, 2010). La institución puede optar por diversas estrategias como hacer promoción y visibilizar el aprendizaje servicio, implementar políticas que den apoyo a los proyectos, formar al profesorado y crear espacios de celebración.

3. Rúbrica de autoevaluación de la calidad de proyectos de aprendizaje servicio en educación superior

A continuación presentamos una herramienta para evaluar las diez características propuestas hasta el momento en el texto. La Rúbrica de autoevaluación de la calidad de proyectos de aprendizaje servicio en educación superior está diseñada para ayudar al profesorado a ajustar y mejorar el desarrollo y el enfoque de los proyectos de aprendizaje servicio que estén llevando a cabo o quieran diseñar. La rúbrica pretende ser un motor de innovación, un factor de reflexión e innovación para la práctica.

La rúbrica pretende ayudar a analizar la calidad de un proyecto en un momento determinado y quiere ser una herramienta que se pueda retomar, tiempo después, para poder evaluar también la evolución del proyecto y el impacto de los cambios que se hayan ido estableciendo. 


\section{Rúbrica de autoevaluación de la calidad de proyectos de aprendizaje servicio en educación superior}

\begin{tabular}{|c|c|c|c|c|c|}
\hline & Nivel 1 & Nivel 2 & Nivel 3 & Nivel 4 & Observaciones \\
\hline $\begin{array}{l}\text { 1. Enfoque de } \\
\text { aprendizaje }\end{array}$ & $\begin{array}{l}\text { El proyecto promueve } \\
\text { aprendizajes basados en la } \\
\text { memorización y el } \\
\text { cumplimiento de unos } \\
\text { requisitos institucionales } \\
\text { sin plantear cambios } \\
\text { actitudinales. }\end{array}$ & $\begin{array}{l}\text { El proyecto desarrolla } \\
\text { aprendizajes que } \\
\text { pretenden que los } \\
\text { estudiantes cambien su } \\
\text { forma de ver el mundo, } \\
\text { sean creadores de su } \\
\text { realidad y se fomenta la } \\
\text { metacognición. }\end{array}$ & $\begin{array}{l}\text { El proyecto desarrolla } \\
\text { aprendizajes que pretenden } \\
\text { que los estudiantes cambien } \\
\text { su forma de ver el mundo, } \\
\text { sean creadores de su } \\
\text { realidad. Además hay un } \\
\text { espacio de reflexión } \\
\text { específico sobre el proyecto. }\end{array}$ & $\begin{array}{l}\text { El proyecto propone además } \\
\text { estrategias de aprendizaje que } \\
\text { están basadas en el interés de } \\
\text { los alumnos por la materia y las } \\
\text { utilizan para maximizar la } \\
\text { comprensión y satisfacer su } \\
\text { curiosidad. Existen espacios } \\
\text { específicos para ello. }\end{array}$ & \\
\hline 2. Competencias & $\begin{array}{l}\text { El proyecto fomenta el } \\
\text { aprendizaje de las } \\
\text { competencias } \\
\text { transversales de: trabajo } \\
\text { colaborativo, habilidades } \\
\text { comunicativas y empatía }\end{array}$ & $\begin{array}{l}\text { El proyecto incide en las } \\
\text { competencias } \\
\text { transversales anteriores y } \\
\text { en la autonomía, la } \\
\text { creatividad, el } \\
\text { pensamiento crítico, la } \\
\text { iniciativa personal y la } \\
\text { sensibilidad } \\
\text { estudiantado. }\end{array}$ & $\begin{array}{l}\text { El proyecto fomenta el } \\
\text { aprendizaje de competencias } \\
\text { profesionales propias de los } \\
\text { estudios del estudiantado. }\end{array}$ & $\begin{array}{l}\text { El proyecto incide en las } \\
\text { competencias curriculares } \\
\text { propias de la asignatura donde } \\
\text { se desarrolla el proyecto. Se } \\
\text { trabajan competencias propias } \\
\text { de la indagación y la } \\
\text { investigación. }\end{array}$ & \\
\hline
\end{tabular}

Campo, L. (2015). Evaluar para mejorar los proyectos de aprendizaje servicio en la universidad. RIDAS, Revista Iberoamericana de Aprendizaje y Servicio, 1, 91-111. DOI 10.1344/RIDAS2015.1.6 


\begin{tabular}{|c|c|c|c|c|}
\hline 3. Nivel de participación & 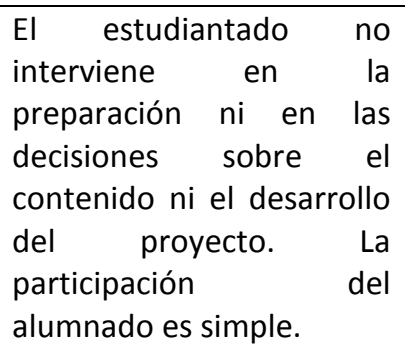 & $\begin{array}{l}\text { El estudiantado puede } \\
\text { opinar y dar su parecer } \\
\text { sobre el proyecto. Se les } \\
\text { anima a opinar o valorar el } \\
\text { proyecto y hay un espacio } \\
\text { determinado para ello. La } \\
\text { participación del } \\
\text { alumnado es consultiva. }\end{array}$ & $\begin{array}{l}\text { El estudiantado participa en } \\
\text { la definición del proyecto, en } \\
\text { la determinación de su } \\
\text { sentido y de sus objetivos. } \\
\text { También participa en el } \\
\text { diseño, la planificación, la } \\
\text { ejecución y la valoración. La } \\
\text { participación es proyectiva. }\end{array}$ & $\begin{array}{l}\text { El estudiantado pide, exige o } \\
\text { genera nuevos espacios y } \\
\text { mecanismos de participación en } \\
\text { el proyecto. Equivale a un nivel } \\
\text { máximo de participación: } \\
\text { metaparticipación }\end{array}$ \\
\hline $\begin{array}{l}\text { 4. Evaluación del } \\
\text { proyecto }\end{array}$ & $\begin{array}{l}\text { Se evalúan los } \\
\text { aprendizajes por parte del } \\
\text { profesor, igual que el } \\
\text { resto de los aprendizajes } \\
\text { curriculares. }\end{array}$ & $\begin{array}{l}\text { Se evalúan los } \\
\text { aprendizajes por parte de } \\
\text { todos los participantes en } \\
\text { el proyecto (responsables } \\
\text { entidad, profesorado y } \\
\text { estudiantes). }\end{array}$ & $\begin{array}{l}\text { Además de los aprendizajes } \\
\text { también es evalúa el servicio } \\
\text { que se ofrece a la } \\
\text { comunidad. }\end{array}$ & $\begin{array}{l}\text { Se evalúan los aprendizajes, el } \\
\text { servicio a la comunidad y el } \\
\text { proyecto de manera global con } \\
\text { el objetivo de mejorarlo en } \\
\text { próximas ediciones. La } \\
\text { evaluación se lleva a cabo por } \\
\text { todos los participantes del } \\
\text { proyecto. }\end{array}$ \\
\hline $\begin{array}{l}5 . \quad \text { Seguimiento } \\
\text { académico en la entidad }\end{array}$ & $\begin{array}{l}\text { No hay seguimiento } \\
\text { académico del alumnado } \\
\text { en la entidad donde se } \\
\text { desarrolla el servicio. }\end{array}$ & $\begin{array}{l}\text { Hay un seguimiento } \\
\text { académico puntual del } \\
\text { alumnado en la entidad } \\
\text { donde se desarrolla el } \\
\text { servicio. }\end{array}$ & $\begin{array}{l}\text { Hay un seguimiento } \\
\text { académico del alumnado en } \\
\text { la entidad coordinado con la } \\
\text { institución formadora. }\end{array}$ & $\begin{array}{l}\text { Hay un seguimiento académico } \\
\text { intenso de los alumnos en la } \\
\text { entidad, coordinado con la } \\
\text { institución formadora. }\end{array}$ \\
\hline 6. Transdisciplinariedad & $\begin{array}{lrr}\text { No se } & \text { propone } & \text { la } \\
\text { posibilidad } & \text { que } \\
\text { estudiantes } & \text { de } & \text { diversos }\end{array}$ & $\begin{array}{l}\text { Estudiantes de diferentes } \\
\text { estudios pero de un } \\
\text { mismo ámbito de }\end{array}$ & $\begin{array}{l}\text { Estudiantes de diferentes } \\
\text { estudios pero de un mismo } \\
\text { ámbito de conocimiento }\end{array}$ & $\begin{array}{lcr}\text { Estudiantes } & \text { de diferentes } \\
\text { estudios y } & \text { ámbitos de } \\
\text { conocimiento } & \text { trabajan sobre }\end{array}$ \\
\hline
\end{tabular}

Campo, L. (2015). Evaluar para mejorar los proyectos de aprendizaje servicio en la universidad. RIDAS, Revista Iberoamericana de Aprendizaje y Servicio, 1, 91-111. DOI 10.1344/RIDAS2015.1.6 


\begin{tabular}{|c|c|c|c|c|}
\hline & estudios trabajen juntos. & $\begin{array}{l}\text { conocimiento trabajan } \\
\text { sobre los mismos retos sin } \\
\text { necesidad } \\
\text { complementarse. }\end{array}$ & $\begin{array}{l}\text { trabajan sobre los mismos } \\
\text { retos y con la necesidad de } \\
\text { complementarse. }\end{array}$ & $\begin{array}{l}\text { los mismos retos con la } \\
\text { necesidad de complementarse. }\end{array}$ \\
\hline $\begin{array}{l}\text { 7. Impacto y proyección } \\
\text { social }\end{array}$ & $\begin{array}{l}\text { El proyecto trabaja sobre } \\
\text { necesidades reales y } \\
\text { cercanas. }\end{array}$ & $\begin{array}{l}\text { El proyecto trabaja sobre } \\
\text { necesidades reales y } \\
\text { cercanas e influye en el } \\
\text { contexto del beneficiario. }\end{array}$ & $\begin{array}{l}\text { El proyecto facilita } \\
\text { herramientas a la comunidad } \\
\text { cuando el proyecto finaliza } \\
\text { (la empodera). }\end{array}$ & $\begin{array}{l}\text { El proyecto influye en la } \\
\text { transformación de la } \\
\text { administración propiciando que } \\
\text { las necesidades sean atendidas } \\
\text { más allá de la ejecución del } \\
\text { proyecto. }\end{array}$ \\
\hline 8. Trabajo en red & $\begin{array}{l}\text { Una institución educativa } \\
\text { y una entidad } \\
\text { forman lazocial } \\
\text { partenariado } r \text { de } \\
\text { construir un proyecto } \\
\text { común. }\end{array}$ & $\begin{array}{l}\text { Una institución educativa } \\
\text { y una entidad social } \\
\text { forman lazos de } \\
\text { partenariado para } \\
\text { construir un proyecto } \\
\text { común con apoyo de } \\
\text { elementos de conexión } \\
\text { institucionalizados. }\end{array}$ & $\begin{array}{l}\text { Una institución educativa y } \\
\text { una entidad social o varias } \\
\text { forman lazos de partenariado } \\
\text { para construir un proyecto } \\
\text { común. Además el proyecto } \\
\text { está conectado a una red de } \\
\text { proyectos similares. }\end{array}$ & $\begin{array}{l}\text { Una institución educativa y una } \\
\text { entidad social o varias forman } \\
\text { lazos de partenariado para } \\
\text { construir un proyecto común. } \\
\text { Además el proyecto está } \\
\text { conectado a una red de } \\
\text { proyectos } \\
\text { institucionalizada similares } \\
\text { intercambiar reflexiones y } \\
\text { mejoras en encuentros } \\
\text { habituales. }\end{array}$ \\
\hline 9. Campo profesional & $\begin{array}{l}\text { El proyecto no modifica la } \\
\text { visión convencional del } \\
\text { campo profesional. }\end{array}$ & $\begin{array}{l}\text { El proyecto contribuye a } \\
\text { abrir una visión del campo } \\
\text { profesional con mayor } \\
\text { implicación social. }\end{array}$ & $\begin{array}{l}\text { El proyecto contribuye a abrir } \\
\text { nuevas visiones profesionales } \\
\text { desder situaciones } \\
\text { organizativas parecidas a las } \\
\text { profesionales con mayor } \\
\text { implicación social. }\end{array}$ & $\begin{array}{l}\text { El proyecto contribuye a abrir } \\
\text { nuevos campos profesionales } \\
\text { con mayor implicación social y } \\
\text { se buscan situaciones } \\
\text { organizativas parecidas a las } \\
\text { profesionales que implica } \\
\text { trabajar con profesionales de } \\
\text { diferentes disciplinas. }\end{array}$ \\
\hline
\end{tabular}

Campo, L. (2015). Evaluar para mejorar los proyectos de aprendizaje servicio en la universidad. RIDAS, Revista Iberoamericana de Aprendizaje y Servicio, 1, 91-111. DOI 10.1344/RIDAS2015.1.6 


\begin{tabular}{|c|c|c|c|c|}
\hline $\begin{array}{l}\text { 10. Institucionalización } \\
\text { académica } \\
\text { 10.1. Difusión }\end{array}$ & $\begin{array}{lr}\text { La institución } & \text { no } \\
\text { promueve } & \text { el } \\
\text { conocimiento } & \text { del } \\
\text { aprendizaje servicio. } & \end{array}$ & $\begin{array}{l}\text { La institución propone } \\
\text { algunas acciones para dar } \\
\text { a conocer el aprendizaje } \\
\text { servicio pero no lo hace de } \\
\text { manera sistemática. }\end{array}$ & $\begin{array}{l}\text { Se facilita la difusión del } \\
\text { aprendizaje servicio entre la } \\
\text { comunidad universitaria. }\end{array}$ & $\begin{array}{l}\text { Se facilita la extensión y } \\
\text { replicación de proyectos de } \\
\text { aprendizaje servicio. }\end{array}$ \\
\hline $\begin{array}{l}\text { 10. Institucionalización } \\
\text { académica } \\
\text { 10.2. Reconocimiento } \\
\text { académico }\end{array}$ & $\begin{array}{l}\text { La institución no muestra } \\
\text { explícitamente su apoyo al } \\
\text { aprendizaje servicio. }\end{array}$ & $\begin{array}{l}\text { La institución muestra } \\
\text { interés por algunos } \\
\text { aspectos de los proyectos } \\
\text { de aprendizaje servicio } \\
\text { pero no de manera } \\
\text { sistematizada. }\end{array}$ & $\begin{array}{l}\text { Los proyectos se ubican en } \\
\text { alguna estructura de la } \\
\text { institución (asignatura, } \\
\text { prácticas o programa). }\end{array}$ & $\begin{array}{l}\text { Existe documentación y } \\
\text { acciones explícitas en la que la } \\
\text { institución muestra su apoyo y } \\
\text { reconocimiento al aprendizaje } \\
\text { servicio. }\end{array}$ \\
\hline $\begin{array}{l}\text { 10. Institucionalización } \\
\text { académica } \\
\begin{array}{l}\text { 10.3. Disponibilidad de } \\
\text { recursos }\end{array}\end{array}$ & $\begin{array}{l}\text { No se facilitan recursos } \\
\text { desde la institución para } \\
\text { llevar a cabo proyecto de } \\
\text { aprendizaje servicio. }\end{array}$ & $\begin{array}{l}\text { Se facilita la organización } \\
\text { del proyecto con } \\
\text { flexibilización de grupos y } \\
\text { horarios en caso de que } \\
\text { sea necesario. }\end{array}$ & $\begin{array}{l}\text { Se facilita la organización del } \\
\text { proyecto con flexibilización } \\
\text { de grupos y horarios en caso } \\
\text { de que sea necesario. } \\
\text { También se facilitan } \\
\text { autorizaciones, acuerdos, } \\
\text { convenios. }\end{array}$ & $\begin{array}{l}\text { Se facilitan recursos, contactos } \\
\text { para hacer red de proyectos, } \\
\text { dispone de un abanico de } \\
\text { servicios posibles. Se facilitan } \\
\text { instrumentos para la evaluación } \\
\text { de los proyectos. Existe una } \\
\text { oficina de coordinación o algún } \\
\text { espacio donde dirigirse. Se } \\
\text { reconoce el tiempo académico } \\
\text { que dedica el profesorado. }\end{array}$ \\
\hline $\begin{array}{l}\text { 10. Institucionalización } \\
\text { académica } \\
\begin{array}{l}\text { 10.4. Relevancia y } \\
\text { visibilidad }\end{array}\end{array}$ & $\begin{array}{l}\text { No existe ningún espacio } \\
\text { de reconocimiento de la } \\
\text { comunidad educativa } \\
\text { hacia el proyecto. }\end{array}$ & $\begin{array}{l}\text { Existe algún reconociendo } \\
\text { pero no está } \\
\text { institucionalizado ni } \\
\text { sistematizado. }\end{array}$ & $\begin{array}{l}\text { Existe un espacio de } \\
\text { reconocimiento y celebración } \\
\text { institucional. }\end{array}$ & $\begin{array}{l}\text { Se favorece el reconocimiento } \\
\text { social a través de premios y } \\
\text { ayudas. }\end{array}$ \\
\hline
\end{tabular}

Cuadro 1: Rúbrica para la evaluación de la calidad de proyectos. Fuente: elaboración propia

Campo, L. (2015). Evaluar para mejorar los proyectos de aprendizaje servicio en la universidad. RIDAS, Revista Iberoamericana de Aprendizaje y Servicio, 1, 91-111. DOI

10.1344/RIDAS2015.1.6 


\section{Conclusiones}

A lo largo del texto hemos propuesto diez elementos que dan calidad a las propuestas de aprendizaje servicio. Consideramos que, por pequeñas que sean, las propuestas de aprendizaje servicio son, en general, buenas propuestas. Éstas pueden transitar por los diez elementos según su complejidad o calidad. Así, por ejemplo, una propuesta puede tener un alto nivel de participación proyectiva de los estudiantes y poca institucionalización pero a su vez, una evaluación completa y rigurosa de todos los aspectos.

Con estas diez categorías hacemos un análisis completo del proyecto de aprendizaje servicio desde la mirada del profesorado y tiendo en cuenta el proyecto como estrategia educativa de docencia y aprendizaje. La elaboración de las diez categorías hace que se haya hecho un esfuerzo importante por valorar qué elementos de calidad son más importantes para el profesorado y simplificando la información para construir una escala de menor a mayor nivel de calidad para cada una de las categorías.

La rúbrica está diseñada para que sea el profesorado quien analice su propio proyecto pero para analizar la calidad de la práctica en su totalidad. Consideramos que el profesorado es la figura clave para evaluar una práctica como propuesta de aprendizaje. En la evaluación del proyecto, también se puede tener en cuenta la voz de los demás participantes de la experiencia: el alumnado; los responsables de la entidad donde se lleva a cabo el servicio, si los hay; y los beneficiarios del servicio que llevan a cabo los estudiantes. En este caso, la herramienta puede ser un instrumento para la discusión compartida sobre la calidad del proyecto entre los diversos agentes participantes en el proyecto. También, se puede usar la rúbrica como instrumento facilitador de la discusión entre expertos o responsables institucionales de programas de aprendizaje servicio. En este sentido, puede ayudar a imaginar algunas estrategias institucionales o valorar hacia dónde hacer esfuerzos para la implementación del aprendizaje servicio en la universidad. Además, la rúbrica puede tener un uso para valorar algunas acciones relacionadas con la responsabilidad social de las universidades y de esta manera, valorar también la calidad de las instituciones universitarias, en cuanto a esta temática. Así, la rúbrica puede ser analizada de manera individual, colectiva o institucional, por figuras de entre iguales o personas con funciones complementarias.

\section{Referencias bibliográficas}

Arratia, A. (2008). Ética, solidaridad y aprendizaje servicio en educación superior. Acta Bioética, 14, 61-67. 


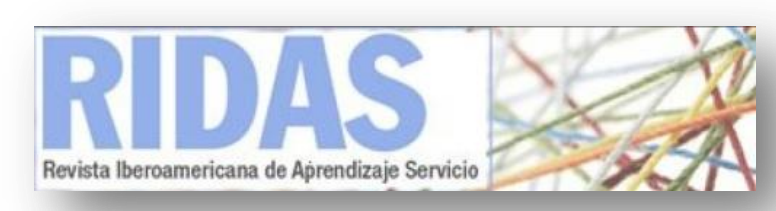

De Miguel, M. (2005). Adaptación de los planes de estudio al proceso de convergencia europea. Oviedo: Ediciones de la Universidad de Oviedo.

Coll, C., Martín, E. \& Onrubia, J. (2001). La evaluación del aprendizaje escolar: dimensiones psicológicas, pedagógicas y sociales. En Coll, C., Palacios, J. y Marchesi, A. Desarrollo psicológico y educación. Madrid: Alianza Editorial.

Cortina, A. (1997). Ciudadanos del mundo. Hacia una teoría de la ciudadanía. Madrid: Alianza Editorial.

Eyler, J. \& Giles, D. (1999). Where's the learning in service-learning? San Francisco: Jossey-Bass Publishers.

Hernández Pina, F., Martínez Clares, P., Da Fonseca, P. \& Rubio, M. (2005). Aprendizaje, competencias y rendimiento en educación superior. Madrid: La Muralla.

Herrero, A. (2010). Una nueva forma de producción de conocimientos: el aprendizajeservicio en educación superior. Tzhoecoen, 5, 63-81. Sipán: Universidad Señor de Sipán.

Jeandron, C. \& Robinson, G. (2010). Creating a Climate for Service Learning Success. Washington, D.C.: American Association of Community Colleges.

Martínez, M. (Eds.) (2008). Aprendizaje servicio y responsabilidad social de las universidades. Barcelona: ICE-Octaedro.

Martínez, M. \& Puig, J.M. (2011). Aprenentatge servei: de l'Escola Nova a l'educació d'avui. Temps d'Educació, 41, 11-24.

Palos, J. (2011). Aprenentatge servei. Aprendre de forma competencial i amb responsabilitat social. Temps d'Educació, 41, 25-40.

Pérez Juste, R. (2006). Evaluación de programas educativos. Madrid: La Muralla

Puig J.M. et. al. (2007). Aprenentatge servei. Educar per a la ciutadania. Barcelona: Octaedro i Fundació Jaume Bofill.

Puig, J.M., Campo, L. \& Climent, T. (2011). Com difondre una innovació perdagògica? EI cas de l'aprenentatge servei a Catalunya. Temps d'Educació, 41. 129-140.

Rubio, L. (2009). El aprendizaje en el aprendizaje servicio. En Puig, J.M. (Coord.), Aprendizaje Servicio (ApS). Educación y compromiso cívico. Barcelona: Graó. 


\section{RDAS}

Tapia, N. (2007). El aprendizaje servicio en las Organizaciones de la Sociedad Civil. En AAVV, Antología 1997-2007. Seminarios Internacionales Aprendizaje y Servicio Solidario. Buenos Aires: MECY.

Recuperado de

https://infanciayjuventudsc.files.wordpress.com/2011/06/antologc3ada-1997-2007-

seminarios-internacionales-aprendizaje-y-servicio-solidario.pdf

Tapia, N. (2008). El aprendizaje servicio en la educación superior. En AAVV, Aprendizaje y servicio solidario en la misión de la Educación Superior. Una mirada analítica desde sus protagonistas. Buenos Aires: MECY.

Recuperado de http://www.bnm.me.gov.ar/giga1/documentos/EL001802.pdf

Tapia, N. (2008). Calidad académica y responsabilidad social: el aprendizaje servicio como puente entre dos culturas universitarias. En Martínez, M. (Eds.), Aprendizaje servicio y responsabilidad social de las universidades. Barcelona: ICE-Octaedro.

Trilla, J. \& Novella, A. (2001). Educación y participación social en la infancia. Revista Iberoamericana de la Educación, 26, 137-164.

Nota final

Las reflexiones principales de este texto son parte de los resultados de la tesis doctoral "Aprendizaje servicio y educación superior. Una rúbrica para evaluar la calidad de proyectos" defendida por la autora en julio de 2014.

\footnotetext{
' Consideraciones expuestas por Furco en la conferencia "Impacto de los proyectos de aprendizaje servicio" en Argentina (2007)
} 